Portland State University

PDXScholar

\title{
Analytically Reduced Form for the Class of Integrals Containing Multicenter Products of 1s Hydrogenic Orbitals, Coulomb or Yukawa Potentials, and Plane Waves
}

Jack C. Straton

Portland State University, straton@pdx.edu

Follow this and additional works at: https://pdxscholar.library.pdx.edu/phy_fac

Part of the Atomic, Molecular and Optical Physics Commons

Let us know how access to this document benefits you.

\section{Citation Details}

Straton, Jack C. "Analytically reduced form for the class of integrals containing multicenter products of $1 \mathrm{~s}$ hydrogenic orbitals, Coulomb or Yukawa potentials, and plane waves." Physical Review A 39 (1989): 5071-5074.

This Article is brought to you for free and open access. It has been accepted for inclusion in Physics Faculty Publications and Presentations by an authorized administrator of PDXScholar. Please contact us if we can make this document more accessible: pdxscholar@pdx.edu. 


\title{
Analytically reduced form for the class of integrals containing multicenter products of $1 s$ hydrogenic orbitals, Coulomb or Yukawa potentials, and plane waves
}

\author{
Jack C. Straton* \\ Laboratory for Astronomy and Solar Physics, Goddard Space Flight Center, \\ National Aeronautics and Space Administration, Greenbelt, Maryland 20771
}

(Received 16 February 1988)

\begin{abstract}
The entire class of integrals containing a product of $N 1 s$ hydrogenic orbitals and $M$ Coulomb or Yukawa potentials (with arguments that are linear combinations of the $m \leq N+M$ coordinates of integration) with $m$ plane waves is analytically reduced from a $3 m$-dimensional integral to a $(M+N-1)$-dimensional integral.
\end{abstract}

\section{INTRODUCTION}

In the preceding paper ${ }^{1}$ the Fourier transform of the multicenter product of $1 s$ hydrogenic orbitals and Coulomb or Yukawa potentials was found. Then the dimensionality of the subsequent integration over plane waves with this product of orbitals and potentials was reduced from $3(m+1)$ to $N+M-1$, where $m \leq N+M$.
The product of orbitals and potentials treated in that paper was not the most general class since they had the Fourier transform coordinate in common. The present paper extends this analytical reduction to the general class of integrals composed of products of plane waves with $1 s$ hydrogenic orbitals and Coulomb or Yukawa potentials in which the arguments are arbitrary linear combinations of the coordinates of integration, ${ }^{2}$

$$
\begin{aligned}
S_{1 s, \ldots, 1 s}^{\lambda_{1}, \ldots, \lambda_{N} ; \eta_{1}, \ldots, \eta_{M}}\left(\mathbf{p}_{1}, \mathbf{p}_{2}, \ldots, \mathbf{p}_{m}\right)= & \int d^{3} x_{1} \cdots d^{3} x_{m} e^{-i\left(\mathbf{p}_{1} \cdot \mathbf{x}_{1}+\cdots+\mathbf{p}_{m} \cdot \mathbf{x}_{m}\right)} \\
& \times u_{1 s}^{\lambda_{1}}\left(\mathbf{R}_{i}\right) \cdots u_{1 s}^{\lambda_{N}}\left(\mathbf{R}_{N}\right) V_{\eta_{1}}\left(\mathbf{R}_{N+1}\right) \cdots V_{\eta_{M}}\left(\mathbf{R}_{N+M}\right),
\end{aligned}
$$

where

$$
\mathbf{R}_{i}=\sum_{j=1}^{m} t_{i j} \mathbf{x}_{j}
$$

The procedure is similar to the previous work, as would be the extensions to excited states noted therein. First one introduces the Feynman representation ${ }^{3}$ for the denominators in the Fourier transforms of the orbitals and potentials to allow addition of all momenta from these denominators within a single denominator. Using an additional integral transformation, this denominator is elevated into an exponential, allowing the momenta in the plane waves to be added in so that all angular dependence lies within a single quadratic form. Then orthogonal transformations are invoked ${ }^{4}$ to diagonalize this quadratic form first with respect to momenta and then with respect to the spatial coordinates. But after integration over momentum and spatial coordinates, the elements of the diagonalized matrices appear only in the form of the determinant of the matrices, which are invariant under the orthogonal transformations, so that these transformations do not need to actually be found. One needs only to calculate the discriminants of the original quadratic forms.

\section{TRANSFORMATIONS AND INTEGRATIONS}

The product of orbitals and potentials is first Fourier transformed using (in atomic units)

$$
u_{1 s}^{\lambda}(\mathbf{R})=\left[\frac{\lambda}{\pi}\right]^{5 / 2} \int d^{3} k \frac{e^{i \mathbf{k} \cdot \mathbf{R}}}{\left(\lambda^{2}+k^{2}\right)^{2}},
$$

where

$$
\lambda=\frac{Z}{a_{0}},
$$

and $^{5}$

$$
V_{\eta}(\mathbf{R})=\frac{e^{-\eta R}}{R}=\frac{1}{2 \pi^{2}} \int d^{3} k \frac{e^{i \mathbf{k} \cdot \mathbf{R}}}{\eta^{2}+k^{2}}, \quad \eta \geq 0 .
$$


Then

$$
\begin{aligned}
S=\left(\frac{\lambda_{1}}{\pi} \frac{\lambda_{2}}{\pi} \cdots \frac{\lambda_{N}}{\pi}\right)^{5 / 2} \frac{1}{\left(2 \pi^{2}\right)^{M}} \int d^{3} x_{1} \cdots d^{3} x_{m} \int d^{3} k_{1} \cdots d^{3} k_{N} d^{3} k_{N+1} \cdots d^{3} k_{N+M} \\
\quad \times \frac{-i\left(\mathbf{p}_{1} \cdot \mathbf{x}_{1}+\cdots+\mathbf{p}_{m} \cdot \mathbf{x}_{m}-\mathbf{k}_{1} \cdot \mathbf{R}_{1}-\cdots-\mathbf{k}_{N} \cdot \mathbf{R}_{N}-\mathbf{k}_{N+1} \cdot \mathbf{R}_{N+1}-\cdots-\mathbf{k}_{N+M} \cdot \mathbf{R}_{N+M}\right)}{\left(\lambda_{1}^{2}+k_{1}^{2}\right)^{2} \cdots\left(\lambda_{N}^{2}+k_{N}^{2}\right)^{2}\left(\eta_{1}^{2}+k_{N+1}^{2}\right) \cdots\left(\eta_{N+1}^{2}+k_{N+M}^{2}\right)} .
\end{aligned}
$$

Note that at this stage one could integrate over the $x$ 's to obtain $\delta$ functions in $m$ of the $N+M$ momentum variables. But sequential integration using these $\delta$ functions becomes increasingly difficult as $N$ and $M$ increase and does not decrease the final number of integrals except in special cases noted in the previous paper, ${ }^{1}$ such as when $m=N+M$.

The next step is to introduce the standard integral transform for the denominators, ${ }^{6}$ generalized to allow arbitrary powers of the denominators,

$$
\frac{1}{D_{1}^{1+m_{1}} D_{2}^{1+m_{2}} \cdots D_{n}^{1+m_{n}}}=\frac{\left(n+\sum_{i} m_{i}-1\right) !}{\left(m_{1}\right) !\left(m_{2}\right) ! \cdots\left(m_{n}\right) !} \int_{0}^{1} d \alpha_{1} \int_{0}^{1} d \alpha_{2} \cdots \int_{0}^{1} d \alpha_{n} \alpha_{1}^{m_{1}} \alpha_{2}^{m_{2}} \cdots \alpha_{n}^{m_{n}} \frac{\delta\left[1-\sum_{i=1}^{n} \alpha_{i}\right]}{\left\lfloor\sum_{i=1}^{n} \alpha_{i} D_{i}\right]^{n+\sum_{i} m_{i}}} .
$$

and, to allow addition of momentum vectors from the plane waves and the denominators, the additional transformation $^{7}$

$$
(v-1) ! D^{-v}=\int_{0}^{\infty} d \rho \rho^{v-1} e^{-\rho D} .
$$

Then (1) can be written in terms of a single quadratic form

$$
\begin{array}{r}
S=\frac{\left[\frac{\lambda_{1}}{\pi} \frac{\lambda_{2}}{\pi} \cdots \frac{\lambda_{N}}{\pi}\right]^{5 / 2} \frac{1}{\left(2 \pi^{2}\right)^{m}}}{\left(m_{1}\right) !\left(m_{2}\right) ! \cdots\left(m_{N}\right) !} \int_{0}^{1} d \alpha_{1} \cdots \int_{0}^{1} d \alpha_{N+M} \alpha_{1} \cdots \alpha_{N} \delta \\
\quad \times \int_{0}^{\infty} d \rho \rho^{2 N+M-1} \int d^{3} x_{1} \cdots d^{3} x_{m} \int d^{3} k_{1} \cdots d^{3} k_{N+M} \alpha^{-\rho Q} \alpha_{i}
\end{array}
$$

where

$$
\begin{aligned}
& Q=\underline{V}^{T} \underline{W} \underline{V}, \\
& \underline{V}^{T}=\left(\mathbf{k}_{1}, \mathbf{k}_{2}, \ldots, \mathbf{k}_{N+M}, 1\right), \\
& \underline{W}=\left[\begin{array}{ccccc}
\alpha_{1} & 0 & \ldots & 0 & \mathbf{b}_{1} \\
0 & \alpha_{2} & & 0 & \mathbf{b}_{2} \\
\vdots & & & \vdots \\
0 & 0 & & \alpha_{N+M} & \mathbf{b}_{N+M} \\
\mathbf{b}_{1} & \mathbf{b}_{2} & \cdots & \mathbf{b}_{N+M} & C
\end{array}\right], \\
& C=\varphi+\frac{i}{\rho} \sum_{i=1}^{m} \mathbf{x}_{i} \cdot \mathbf{p}_{i}, \\
& \varphi=\sum_{i=1}^{N} \lambda_{i}^{2} \alpha_{i}+\sum_{i=N+1}^{N+M} \eta_{i}^{2} \alpha_{i},
\end{aligned}
$$

and

$$
\mathbf{b}_{j}=-\frac{i}{2 \rho} \sum_{i=1}^{m} t_{j i} \mathbf{x}_{i}
$$

By an orthogonal transformation and a simple translation in $\left\{\mathbf{k}_{1}, \mathbf{k}_{2}, \ldots, \mathbf{k}_{N}\right\}$ space (with Jacobian $=1$ ), $Q$ may be reduced to diagonal form

$Q^{\prime}=a_{1}^{\prime} k_{1}^{\prime 2}+a_{2}^{\prime} k_{2}^{\prime 2}+\cdots+a_{N+M}^{\prime} k_{N+M}^{\prime 2}+c^{\prime}$,

allowing the $k$ integrals to be done, $\int d^{3} k_{1}^{\prime} \cdots d^{3} k_{N+M}^{\prime} \exp \left[-\rho\left(a_{1}^{\prime} k_{1}^{\prime 2}+\cdots+a_{N+M}^{\prime} k_{N+M}^{\prime 2}\right)\right]$

$$
=\left(\frac{\pi^{N+M}}{\rho^{N+M} \Lambda}\right)^{3 / 2},
$$

where the invariant determinant $\Lambda$ is

$$
\begin{aligned}
\Lambda & =\left|\begin{array}{cccc}
\alpha_{1} & 0 & \cdots & 0 \\
0 & \alpha_{2} & & 0 \\
\vdots & & & \vdots \\
0 & 0 & \cdots & \alpha_{N+M}
\end{array}\right| \\
& =\prod_{i=1}^{N+M} \alpha_{i} \\
& =\prod_{i=1}^{N+M} a_{i}^{\prime} .
\end{aligned}
$$

To integrate over the $x$ 's, $c^{\prime}\left(x_{j}\right)$ must be determined. The discriminant of the quadratic form, which is invariant under the transformation, is given by $\Omega=\operatorname{det} \underline{W}=\Lambda c^{\prime}$

$$
\begin{aligned}
& =C \Lambda+\sum_{i=1}^{N+M} \sum_{j=1}^{N+M} \mathbf{b}_{i} \cdot \mathbf{b}_{j}(-1)^{i+j+1} \Lambda_{i j} \\
& =C \Lambda-\sum_{i=1}^{N+M} b_{i}^{2} \prod_{j \neq i}^{N+M} \alpha_{j}
\end{aligned}
$$

where the minor $\Lambda_{i j}$ is $\Lambda$ with the $i$ th row and $j$ th column deleted, 


$$
\Lambda_{i j}=\prod_{l \neq i, j}^{N+M} \alpha_{l}=\delta_{i j} \prod_{l \neq i}^{N+M} \alpha_{l} .
$$

Consider the last line of (19). Gathering the $x$-dependent parts, from (13) and (15),

$$
\Omega=\varphi \Lambda+H \text {, }
$$

where

$$
\begin{aligned}
& H=-\underline{X}^{T} \underline{Z} \underline{X}, \\
& \underline{X}^{T}=\left(\begin{array}{cccc}
i \frac{\mathbf{x}_{1}}{2 \rho}, i \frac{\mathbf{x}_{2}}{2 \rho} \ldots, i \frac{\mathbf{x}_{m}}{2 \rho},-1
\end{array}\right), \\
& \underline{Z}=\left|\begin{array}{ccccc}
z_{11} & z_{12} & \ldots & z_{1 m} & \Lambda \mathbf{p}_{1} \\
z_{21} & z_{22} & & z_{2 m} & \Lambda \mathbf{p}_{2} \\
\vdots & & & & \vdots \\
z_{m 1} & z_{m 2} & & z_{m m} & \Lambda \mathbf{p}_{m} \\
\Lambda \mathbf{p}_{1} & \Lambda \mathbf{p}_{2} & \cdots & \Lambda \mathbf{p}_{m} & 0
\end{array}\right|,
\end{aligned}
$$

and

$$
z_{i j}=\sum_{i^{\prime}=1}^{N+M} t_{i^{\prime} i} t_{i^{\prime} j} \prod_{k \neq i^{\prime}}^{N+M} \alpha_{k} .
$$

As with (16), one may use an orthogonal transformation and a translation in $\left\{\mathbf{x}_{1}, \mathbf{x}_{2}, \ldots, \mathbf{x}_{m}\right\}$ space to reduce $H$ to diagonal form,

$$
H^{\prime}=\frac{z_{1}^{\prime}}{4 \rho^{2}} x_{1}^{\prime 2}+\frac{z_{2}^{\prime}}{4 \rho^{2}} x_{2}^{\prime 2}+\cdots+\frac{z_{m}^{\prime}}{4 \rho^{2}} x_{m}^{\prime 2}+g^{\prime},
$$

where $g^{\prime}$, the quadratic form in the external momenta $\mathbf{p}_{j}$, and $z_{i}^{\prime}$ are non-negative. Defining the invariant determinant

$$
z_{1}^{\prime} z_{2}^{\prime} \cdots z_{m}^{\prime}=\Delta \equiv\left|\begin{array}{cccc}
z_{11} & z_{12} & \cdots & z_{1 m} \\
z_{21} & z_{22} & & z_{2 m} \\
\vdots & & & \vdots \\
z_{m 1} & z_{m 2} & \cdots & z_{m m}
\end{array}\right|
$$

allows the final term of (26) to be written

$$
g^{\prime}=-\xi / \Delta
$$

where $\zeta$ is the invariant determinant

$$
\zeta \equiv|\underline{Z}|=\Lambda^{2} \sum_{i=1}^{m} \sum_{j=1}^{m} \mathbf{p}_{i} \cdot \mathbf{p}_{j}(-1)^{i+j+1} \Delta_{i j} .
$$

Then, from (19) and (21),

$$
c^{\prime}=(\varphi \Lambda+H) / \Lambda \text {, }
$$

so that, from (26) and (28),

$$
\begin{aligned}
\rho c^{\prime}= & \rho\left[\varphi-\frac{\zeta}{\Delta \Lambda}\right]+\frac{z_{1}^{\prime}}{4 \rho \Lambda} x_{1}^{\prime 2}+\frac{z_{2}^{\prime}}{4 \rho \Lambda} x_{2}^{\prime 2}+\cdots \\
& +\frac{z_{m}^{\prime}}{4 \rho \Lambda} x_{m}^{\prime 2} .
\end{aligned}
$$

Then the $x^{\prime}$ integrals of (9) are given by

$$
\begin{array}{r}
\int d^{3} x_{1}^{\prime} \cdots d^{3} x_{m}^{\prime} \exp \left[-\left[\frac{z_{1}^{\prime}}{4 \rho \Lambda} x_{1}^{\prime 2}+\frac{z_{2}^{\prime}}{4 \rho \Lambda} x_{2}^{\prime 2}\right.\right. \\
\left.\left.+\cdots+\frac{z_{m}^{\prime}}{4 \rho \Lambda} x_{m}^{\prime 2}\right]\right] \\
=\left[\frac{(4 \rho \Lambda \pi)^{m}}{\Delta}\right]^{3 / 2},
\end{array}
$$

and the $\rho$ integral is, from (8),

$$
\begin{array}{r}
\int_{0}^{\infty} d \rho \rho^{2 N+M-1-3(N+M) / 2+3 m / 2} e^{-\rho(\varphi-\zeta /(\Delta \Lambda))} \\
=\frac{\Gamma((3 m+N-M) / 2)}{[\varphi-\zeta /(\Delta \Lambda)]^{(3 m+N-M) / 2}} .
\end{array}
$$

Then the final result is

$$
\begin{aligned}
& S_{1 s, \ldots, 1 s}^{\lambda_{1}, \ldots, \lambda_{N} ; \eta_{1}, \ldots, \eta_{M}}\left(\mathbf{p}_{1}, \mathbf{p}_{2}, \ldots, \mathbf{p}_{m}\right) \\
& =\frac{\left(\lambda_{1} \cdots \lambda_{N}\right)^{5 / 2} 2^{3 m-M} \Gamma((3 m+N-M) / 2)}{\pi^{N+M / 2-3 m / 2}} \\
& \quad \times \int_{0}^{1} d \alpha_{1} \cdots \int_{0}^{1} d \alpha_{N+M} \alpha_{1} \cdots \alpha_{N} \delta\left[1-\sum_{i=1}^{N+M} \alpha_{i}\right] \frac{\Lambda^{3 m+(N-M-3) / 2} \Delta^{(3 m+N-M-3) / 2}}{(\varphi \Lambda \Delta-\zeta)^{(3 m+N-M) / 2}}
\end{aligned}
$$

Since this expression involves only the invariant determinants, $\Lambda, \Delta$, and $\zeta$, rather than the individual coefficients in the diagonalized quadratic forms (16) and (26), the orthogonal transformations leading to these diagonal forms do not need to be explicitly calculated.

\section{CONCLUSION}

The present approach has analytically reduced the dimensionality of the entire class of integrals containing the multicenter product of plane waves with $1 s$ hydrogenic and Coulomb or Yukawa potentials. The difficult calculus coupled to algebraic manipulation that is necessary for reducing the dimensionality of integrals in a given problem has been done 
once and for all so that time may instead be spent on the development and physical meaning of theories. The reader is directed to Ref. 1 for extensions necessary to include excited states and for the case in which all $\mathbf{R}_{i}$ have some $\mathbf{x}_{j}$ in common, allowing a $3(m+1)$-dimensional integral to be reduced to $N+M-1$ dimensions. $^{8}$

\section{ACKNOWLEDGMENTS}

This work was done while the author was a great scientist conducting research through the National Research Council-NASA Research Associateship Program. Computer costs were funded by NASA-RTOP Grant No. 681-17010-01-08.

*Present address: Department of Physics, Cardwell Hall, Kansas State University, Manhattan, KS 66502.

${ }^{1}$ Jack C. Straton, preceding paper, Phys. Rev. A 39, 5062 (1989).

${ }^{2}$ The reader is cautioned to combine any products that have identical $R$ 's into a single term (by adding the $\lambda$ 's and/or $\eta$ 's) because the final number of integrals depends on the initial number of products.

${ }^{3}$ R. P. Feynman, Phys. Rev. 76, 769 (1949).

${ }^{4}$ J. S. R. Chisholm, Proc. Cambridge Phil. Soc. 48, 300 (1952).

${ }^{5}$ H. B. Dwight, Tables of Integrals and other Mathematical Data (Macmillan, New York, 1961), p. 225, Eq. 859.004. For the case $\eta=0$ this transform is defined only in the sense of a distribution.
${ }^{6} \mathrm{~S}$. S. Schweber, An Introduction to Quantum Field Theory (Harper and Row, New York, 1962), p. 913.

${ }^{7}$ I. S. Gradshteyn and I. M. Ryzhik, Table of Integrals, Series, and Products (Academic, New York, 1980), p. 317, Eq. 3.381(4).

${ }^{8}$ There is an additional class of integrals in which the $R$ 's of (2) include coordinate vectors, in addition to the $x$ 's, that will not be integrated over. The reader is directed to Jack C. Straton, Phys. Rev. A 39, 1676 (1989), for the reduced form of these integrals. The present result also holds for such integrals if the additional terms, appearing in (2), (13), and (24), are added to $\zeta$ of (29). 